\title{
Streptokokken-Pharyngitis
}

\section{Gute Erfahrungen mit Cistusextrakt}

In unserer "Sprechstunde Naturheilkunde" gab Frau Prof. Karin Kraft, Rostock, in MMW Nr. 32-34/2011, S. 18 Tipps zur Behandlung der Tonsillitis. Dazu ein Leser:

- Ohne Frage wäre eine leitliniengerechte Behandlung einer suppurativen Streptokokken-Pharyngitis/-Tonsillitis wünschenswert, wenn denn die Leitlinien wissenschaftliche Evidenz nachvollziehbar zum Ausdruck bringen würden. Ein internationaler Leitlinienvergleich zeigt jedoch sehr viel mehr Unterschiede der Empfehlungen als die einzige Gemeinsamkeit, primär ein Schmalspektrumantibiotikum zur Eradikation einer nachgewiesenen Streptokokkeninfektion einzusetzen [1]. Trotzdem wird die eitrige Mandelentzündung bei Kindern und Heranwachsenden nicht nur in naturheilkundlichen Kreisen - oft als gutartige, selbstlimitierende Erkrankung aufgefasst, bei der Antibiotika nur ausgewählten Fällen vorbehalten bleiben sollten [2].

Auf Grundlage eigener Erfahrungen, z. B. mit fünf eigenen Kindern, bei denen es trotz Antibiose zu immer häufigeren Tonsillitisrezidiven mit zunehmend schwereren, fiebrigen Verläufen kam, haben wir uns vor Jahren für eine naturheilkundliche Behandlung entschieden. Eine solche Rezidivierung bzw. Chronifizierung der Streptokokken-Angina drückt die ärztliche Erfahrung aus, dass ein gutes Drittel aller Antibiotika-Therapien bei dieser Indikation versagen - ein therapeutisches Dilemma [3]. Hinzu kommt, dass ein ebenfalls gutes Drittel aller Tonsillopharyngitiden viraler Genese sind [4].

Entsprechend unserer Forschungen zu den antimikrobiellen Wirkungen einer Unterart der graubehaarten Zistrose haben wir diese, im Mittelmeerraum auch bei Schleimhautentzündungen traditionell verwendete, Arzneipflanze ausgewählt [5], zunächst in Form eines mit-

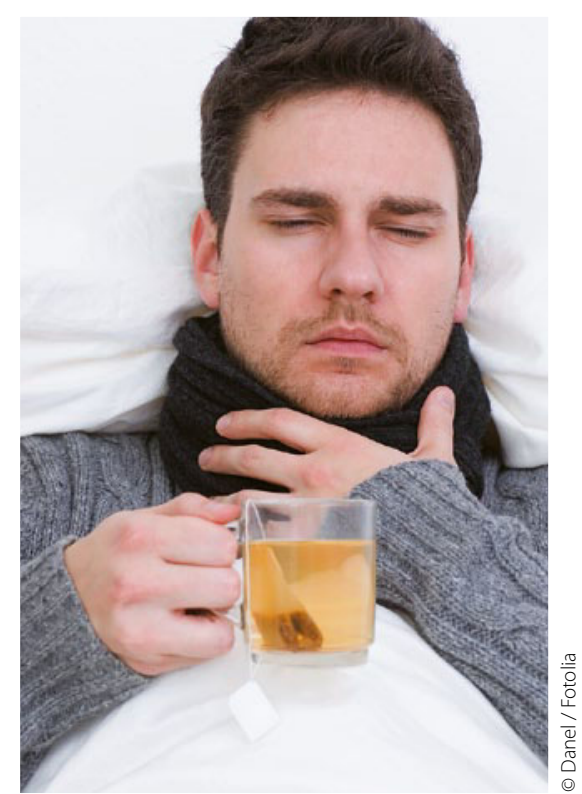

Braucht er Antibiotika oder reicht Tee?

tels Sprühflasche im Rachenraum lokal applizierten Dekoktes. Diese Applikation erwies sich jedoch weniger wirksam als das mehrfach tägliche Gurgeln mit dem Cistusextrakt. Schon einen Tag nach dem Behandlungsbeginn verschwand das Fieber, zwei Tage später der Großteil der eitrigen Stippchen. In der Folgezeit traten immer seltener Rezidive auf.

Diese empirischen Erfahrungen konnten später im Rahmen einer klinischen Studie bestätigt werden [6]. Dass unser Extrakt aus der Varietät Cistus incanus ssp. PANDALIS - Cystus052 - in vitro auch gegen betahämolysierende Streptokokken hoch wirksam ist, konnte u. a. im Agardiffusionstest nachgewiesen werden [Daten unveröffentlicht]. Einer der Wirkmechanismus von Cystustee - die effektive Blockade der mikrobiellen Adhäsion - konnte erst kürzlich wieder bei einer Untersuchung von oropharyngealen Pathogenen, einschließlich Streptokokken, bestätigt werden [7].

\section{Literatur beim Verfasser}

- Dr. Georgios Pandalis, Füchtenweg 3, D-49219 Glandorf

\section{Akupressur mittels Armband}

\section{Keine ausreichende Stimulation}

Die Akupressur mittels Armband hilft nicht gegen Erbrechen während der Wehen und unter der Geburt, berichtete Prof. E. Ernst, Exeter, in MMW Nr. 26-28/2011, S. 26. Dazu ein Leser:

- Ich persönlich würde bezweifeln, dass ein Armband, selbst bei korrekter Punktlokalisation, während des Geburtsvorganges an seiner ursprünglichen Stelle verbleibt. Ich bezweifle auch, dass ein rein statischer Druck eine ausreichende Punktstimulation im Sinne der Akupressur bewirken kann. Unter Umständen wurde also hier Teilplacebo mit Teilplacebo verglichen. Interessant wäre, die Inzidenz ohne Armband zu kennen.

- Dr. med. Volker Isak, Sommerauerstraße 51 a, D-78112 St. Georgen 